\title{
The decay of Hill's vortex in a rotating flow
}

\author{
Matthew N. Crowe, Cameron J. D. Kemp and Edward R. Johnson
}

Department of Mathematics, University College London, London, WC1E 6BT, UK

(Received $\mathrm{xx}$; revised $\mathrm{xx}$; accepted $\mathrm{xx}$ )

Hill's vortex is a classical solution of the incompressible Euler equations which consists of an axisymmetric spherical region of constant vorticity matched to an irrotational external flow. This solution has been shown to be a member of a one-parameter family of steady vortex rings and as such is commonly used as a simple analytic model for a vortex ring. Here we model the decay of a Hill's vortex in a weakly rotating flow due to the radiation of inertial waves. We derive analytic results for the modification of the vortex structure by rotational effects and the generated wave-field using an asymptotic approach where the rotation rate, or inverse Rossby number, is taken to be small. Using this model, we predict the decay of the vortex speed and radius by combining the flux of vortex energy to the wave-field with the conservation of peak vorticity. We test our results against numerical simulations of the full axisymmetric Navier-Stokes equations.

\section{Introduction}

Hill's vortex (Hill 1894) is a classical axisymmetric solution of the incompressible Euler equations in which a rotational spherical vortex is joined to an irrotational far field flow. The far field flow was shown to match the flow around a sphere hence the vortex resembles a travelling point dipole when viewed from far away. This solution has been shown to correspond to an extreme member of a one-parameter family of steady vortex rings (Fraenkel 1972; Norbury 1972, 1973) and is commonly used as a simple analytic model for a vortex ring. Moffatt (1969) showed that there exists a doubly infinite family of spherical vortices where the Hill's vortex solution corresponds to the special case of vanishing azimuthal velocity. Moffatt \& Moore (1978) showed that the Hill's vortex solution is linearly stable to small axisymmetric disturbances and a perturbed vortex would either entrain or detrain fluid from the rear stagnation point to return to a spherical shape. However, the perturbation was shown to be swept down the surface of the vortex to the rear stagnation point where perturbations could grow and potentially lead to a breakdown of the vortex once nonlinear effects became important (Pozrikidis 1986; Protas \& Elcrat 2016).

When the system is rotated, the fluid admits inertial wave solutions so it could be expected that a vortex in a rotating fluid would generate an inertial wave wake in a similar manner to a solid body. This wave generation by solid bodies has been extensively studied both experimentally (Taylor 1922; Long 1953; Machicoane et al. 2018) and analytically (Taylor 1922; Fraenkel 1956; Bretherton 1967) and a general theory for the generation of dispersive waves by moving forcing is given by Lighthill (1967). The interaction between Hill's vortex and an incident wave-field was examined by Llewellyn Smith \& Ford (2001) as an example of acoustic scattering however the generation of waves by Hill's vortex has not been extensively studied.

Scase \& Terry (2018) noted that the steady solutions postulated by Taylor (1922) can be matched to the Hill's vortex solution. A conservation of angular momentum argument led Taylor (1922) to believe that that those of his solutions with closed streamlines could 
not arise in an initial value problem. Appendix B shows, additionally, that the form of solution chosen by Taylor immediately implies energy input from infinity and hence does not satisfy the radiation condition on flows driven by compact forcing.

Steady wave generation problems assume that energy is injected into the system at a constant rate by the forcing term (Lighthill 1967). In the case of waves generated by a vortex, however, the vortex energy will decrease as energy is transferred to the wave-field which in turn leads to a weaker wave forcing. This results in a feedback mechanism in which the vortex decays due to an outward flux of wave energy (Flierl \& Haines 1994; Johnson \& Crowe 2021). If the timescale of this decay is sufficiently long, we may use a timescale separation approach where the problem is assumed to be steady over the short inertial timescale and vary over the slow decay timescale which is set by the outward energy flux. This may be viewed as a slow-manifold approach similar to that used by (Ford et al. 2000) to examine the emission of waves by a quasi-geostrophic vortex. Flierl \& Haines (1994) used this method to examine the decay of a beta-plane modon due to Rossby wave emission. They assumed that the modon structure remained the same throughout the evolution and hence the energy of the modon could be determined using only its speed and radius. By combining the loss of vortex energy to the wavefield with the observation that the peak potential vorticity remained constant throughout the evolution, the slow evolution of the modon speed and radius could be determined. A similar method was also used in Johnson \& Crowe (2021) to examine the decay of a cylindrical vortex in both stratified flow and rotating flow.

Here we model the decay of Hill's vortex by inertial wave radiation in the limit of weak rotation (corresponding to large Rossby number) using the approach of Flierl \& Haines (1994) and Johnson \& Crowe (2021). We begin by determining the leading order Hill's vortex solution modified by weak rotation in terms of the vortex speed and radius. The inner solution is then matched to a linear wave-field far from the vortex and the wavefield is used to determine the energy flux out of the vortex. This approach is effective as there exists a 'Long's model' for this geometry, i.e. a linear integral of the full nonlinear equations of motion. The wave-field satisfies this nonlinear system and so is a valid solution near the vortex (where the amplitude is not small) with only small modifications due to the finite vortex size. Combining an energy balance with the conservation of peak vorticity allows us to predict the evolution of the vortex radius and speed in the weak rotation limit. Finally our predictions are tested against numerical simulations performed using Dedalus (Burns et al. 2020) and the limitations of our model are discussed.

\section{Setup and governing equations}

We begin by introducing Cartesian axes, $O x y z^{\prime}$, describing a frame rotating around the vertical $\left(z^{\prime}\right)$ axis with angular velocity $\Omega$. We consider the evolution of a spherical vortex of (spherical) radius $a(t)$ moving with speed $U(t)$ in the $z^{\prime}$ direction so we define a new vertical coordinate

$$
z=z^{\prime}-\int_{0}^{t} U\left(t^{\prime}\right) \mathrm{d} t^{\prime},
$$

such that the coordinate origin moves with the vortex. This transformation is only applied to coordinates and not to the associated velocity fields. Our nondimensional system is 


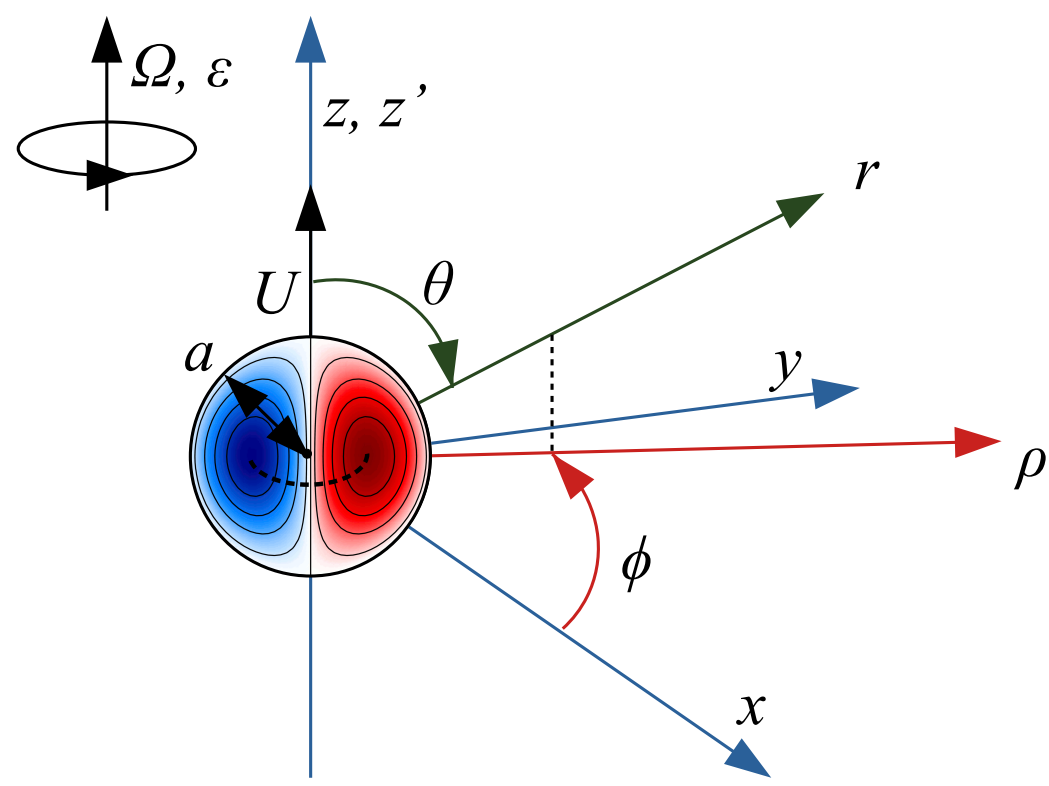

(a)

Figure 1: Diagram of our setup showing Cartesian coordinates, $(x, y, z)$, cylindrical radius, $\rho$, azimuthal angle, $\phi$, spherical radius, $r$ and polar angle, $\theta$. A spherical vortex of radius $a(t)$ moves vertically with velocity $U(t)$ and the system is rotating with angular velocity of $\Omega$ corresponding to an inverse Rossby number of $\epsilon$.

described by the rotating, incompressible Euler equations

$$
\begin{aligned}
{\left[\frac{\partial}{\partial t}-U \frac{\partial}{\partial z}\right] \mathbf{u}+(\mathbf{u} \cdot \nabla) \mathbf{u}+\epsilon \hat{\boldsymbol{z}} \times \mathbf{u} } & =-\nabla p, \\
\nabla \cdot \mathbf{u} & =0,
\end{aligned}
$$

where we note that all velocities are relative to the background fluid and we do not gain any fictitious forces beyond the Coriolis force (Johnson \& Crowe 2021). Here we have nondimensionalised velocities by $V$, lengths by $L$, time by $L / V$ and pressure by $\rho_{0} V^{2}$ for density $\rho_{0}$. The length scale $L$ and velocity scale $V$ are set by the initial size and translational velocity of the vortex. The strength of the rotation is represented in our nondimensional equations by the inverse Rossby number, defined as

$$
\epsilon=\frac{1}{R o}=\frac{2 \Omega L}{V}
$$

which will be taken to be a small parameter. A diagram of our system is given in Fig. 1.

We now take the coordinate origin to be the centre of the spherical vortex and introduce cylindrical coordinates $(\rho, \phi, z)$ where the cylindrical radius is given by $\rho^{2}=x^{2}+y^{2}$ and the azimuthal angle $\tan \phi=y / x$. Under the assumption that the flow is independent of the azimuthal angle, $\phi$, the velocity field may be written as

$$
\mathbf{u}(\rho, z)=u_{\rho} \hat{\boldsymbol{\rho}}+w \hat{\boldsymbol{\phi}}+u_{z} \hat{\boldsymbol{z}}=-\frac{1}{\rho} \frac{\partial \psi}{\partial z} \hat{\boldsymbol{\rho}}+w \hat{\boldsymbol{\phi}}+\frac{1}{\rho} \frac{\partial \psi}{\partial \rho} \hat{\boldsymbol{z}}
$$

for Stokes streamfunction $\psi$ and swirl velocity $w$. We will later use spherical polar 
coordinates $(r, \theta, \phi)$ when solving for the vortex. Here the spherical radius $r$ is defined by $r^{2}=\rho^{2}+z^{2}$ and the polar angle by $\tan \theta=\rho / z$.

As we will show later, there are two relevant timescales in the problem. The first is the transient timescale on which the wave field develops and the second is the slow evolution timescale on which the vortex loses energy. We therefore expand our time derivative using a multiple scales approach to write

$$
\frac{\partial}{\partial t} \rightarrow \frac{\partial}{\partial t}+\delta \frac{\partial}{\partial T}
$$

where $T=\delta t$ is the slow evolution timescale and $\delta$ is a small parameter. It will be shown later that $\delta=\epsilon^{4}$. Finally we will ignore the problem of the transient evolution and look for solutions with a fully developed wave field. Therefore we take $\partial / \partial t=0$ and consider only the slow evolution on timescale $T$. Since the vortex speed and radius may slowly evolve as energy is lost we take $U=U(T)$ and $a=a(T)$.

\subsection{The vorticity equation}

Taking the curl of Eq. (2.2a) and neglecting the transient evolution gives the vorticity equation

$$
\left[\delta \frac{\partial}{\partial T}-U \frac{\partial}{\partial z}\right] \boldsymbol{\omega}+\nabla \times(\boldsymbol{\omega} \times \mathbf{u})-\epsilon \frac{\partial \mathbf{u}}{\partial z}=0,
$$

for vorticity

$$
\boldsymbol{\omega}=-\frac{1}{\rho} \frac{\partial}{\partial z}(\rho w) \hat{\boldsymbol{\rho}}-\frac{1}{\rho} D^{2} \psi \hat{\boldsymbol{\phi}}+\frac{1}{\rho} \frac{\partial}{\partial \rho}(\rho w) \hat{\boldsymbol{z}} .
$$

Here $D^{2}$ is related to the Laplacian operator and is given by

$$
D^{2} f=\nabla^{2} f-\frac{2}{\rho} \frac{\partial f}{\partial \rho}=\rho \frac{\partial}{\partial \rho}\left(\frac{1}{\rho} \frac{\partial f}{\partial \rho}\right)+\frac{\partial^{2} f}{\partial z^{2}} .
$$

Substituting Eqs. (2.4) and (2.7) into Eq. (2.6) gives two equations for $\psi$ and $w$ from the three vorticity components. Both the $\rho$ and $z$ components of the vorticity equation give

$$
\left[\delta \frac{\partial}{\partial T}-U \frac{\partial}{\partial z}\right](\rho w)+\frac{1}{\rho} J[\psi, \rho w]-\epsilon \frac{\partial \psi}{\partial z}=0
$$

while the $\phi$ component gives

$$
\left[\delta \frac{\partial}{\partial T}-U \frac{\partial}{\partial z}\right]\left[\frac{1}{\rho} D^{2} \psi\right]+J\left[\psi, \frac{1}{\rho^{2}} D^{2} \psi\right]+\frac{1}{\rho^{3}} \frac{\partial}{\partial z}(\rho w)^{2}+\epsilon \frac{\partial w}{\partial z}=0 .
$$

The Jacobian operator $J$ is taken to be between $\rho$ and $z$ hence

$$
J[f, g]=\frac{\partial(f, g)}{\partial(\rho, z)}=\frac{\partial f}{\partial \rho} \frac{\partial g}{\partial z}-\frac{\partial f}{\partial z} \frac{\partial g}{\partial \rho} .
$$

The rotation terms in Eqs. (2.9) and (2.10) may now be combined with the swirl velocity and the $U \partial_{z}$ terms may be written using the Jacobian derivative to give

$$
\delta \frac{\partial}{\partial T} \Gamma+\frac{1}{\rho} J\left[\psi-\frac{U}{2} \rho^{2}, \Gamma\right]=0
$$

and

$$
\delta \frac{\partial}{\partial T}\left[\frac{1}{\rho} D^{2} \psi\right]+J\left[\psi-\frac{U}{2} \rho^{2}, \frac{1}{\rho^{2}} D^{2} \psi\right]+\frac{1}{\rho^{3}} \frac{\partial}{\partial z} \Gamma^{2}=0,
$$


where

$$
\Gamma=\rho w+\frac{\epsilon \rho^{2}}{2},
$$

is the total azimuthal circulation divided by $2 \pi$. Eqs. (2.12) and (2.13) are our governing equations which will be used throughout this analysis. On the $z$ axis $(\rho=0)$ we impose the condition that $w=0$ and $\psi=0$ such that all components of velocity and vorticity are finite and continuous.

\subsection{Asymptotic approach}

To proceed we will assume that $\delta$ is small and expand $\psi$ and $w$ using a regular perturbation expansion of the form

$$
(\psi, w)=\left(\psi_{0}, w_{0}\right)+\delta\left(\psi_{1}, w_{1}\right)+O\left(\delta^{2}\right),
$$

where each term in the expansion is a function of $\epsilon$. We will later show that $\epsilon$ can be related to $\delta$ through $\epsilon^{4}=\delta$ and at this point we could expand each term in powers of $\epsilon=\delta^{1 / 4}$ before reordering terms such that the expansion is asymptotically we-ordered in $\delta$. Anticipating the relation $\epsilon^{4}=\delta$, we therefore ignore any components which are $O\left(\epsilon^{4}\right)$ or higher when calculating the leading order solution in $\delta$.

To leading order in $\delta$ Eqs. (2.12) and (2.13) give that

$$
\frac{1}{\rho} J\left[\psi_{0}-\frac{U}{2} \rho^{2}, \rho w_{0}+\frac{\epsilon \rho^{2}}{2}\right]=0,
$$

and

$$
J\left[\psi_{0}-\frac{U}{2} \rho^{2}, \frac{1}{\rho^{2}} D^{2} \psi_{0}\right]+\frac{1}{\rho^{3}} \frac{\partial}{\partial z}\left(\rho w_{0}+\frac{\epsilon \rho^{2}}{2}\right)^{2}=0 .
$$

We begin by using Eq. (2.16) to relate the swirl velocity, $w$, to $\psi$.

\subsection{The swirl velocity and Kelvin's circulation theorem}

Eq. (2.12) states that the azimuthal circulation is conserved following a ring of particles of radius $\rho$, where the term $\epsilon \rho^{2} / 2$ describes the circulation of the rotating frame. This can be thought of as a consequence of Noether's theorem: axial angular momentum in conserved due to the invariance of the system to azimuthal rotation. Consider an incoming ring of particles that far from the vortex is arbitrarily close to the $z$ axis, i.e. $\rho \ll 1$, so $\Gamma$ is vanishingly small. The circulation $\Gamma$ is conserved as the ring of particles expands to pass around the vortex and so $\Gamma$ remains arbitrarily small arbitrarily close to the vortex with $\Gamma$ vanishing on the vortex boundary by continuity. Thus

$$
w_{0}=-\frac{\epsilon \rho}{2} \quad \text { at } \quad r=a .
$$

Eq. (2.16) implies that

$$
\rho w_{0}+\frac{\epsilon \rho^{2}}{2}=K\left(\psi_{0}-\frac{U}{2} \rho^{2}\right),
$$

for some function $K=K(\varphi)$. This result is simply Kelvin's circulation theorem for a ring of particles an axisymmetric flow. We now take

$$
\psi_{0}=\frac{U}{2} \rho^{2} \quad \text { at } \quad r=a,
$$

and use Eq. (2.18) to impose the condition that $K(0)=0$. We note that Eq. (2.20) is 
equivalent to the requirement that the vortex boundary, $r=a$, is a streamsurface in a frame moving with the vortex.

For the fluid outside the spherical vortex, all streamlines must originate from the ultra far-field where $\psi_{0} \rightarrow 0$ and $w_{0} \rightarrow 0$. Therefore Eq. (2.19) gives that

$$
\frac{1}{2} \epsilon \rho^{2}=K\left(-\frac{U}{2} \rho^{2}\right)
$$

hence, outside the vortex, we can determine $K$ as the linear function

$$
K(\varphi)=-\epsilon k \varphi,
$$

for some field $\varphi$. Here we have defined $k(T)=1 / U(T)$ to be an effective wavenumber.

Inside the vortex Eq. (2.19) remains valid, however as streamlines do not necessarily originate from the far field, we are unable to determine $K$ using a far field condition. The choice of this function $K$ is arbitrary and picking a general linear function, $K(\varphi)=\alpha \varphi$, for some parameter $\alpha$ results in the family of vortices considered by (Moffatt 1969). To ensure that our vortex reduces to Hill's vortex as $\epsilon \rightarrow 0$, we assume that Eq. (2.22) continues to hold within the vortex. This assumption corresponds to a vortex with a slow swirl and ensures that $\partial w_{0} / \partial r$ is continuous across $r=a$. The value of $\alpha=-\epsilon k$ can be thought of as an internal wavenumber describing the azimuthal rotation rate of the vortex.

\subsection{The streamfunction}

Using Eq. (2.19), Eq. (2.17) becomes

$$
J\left[\psi_{0}-\frac{U}{2} \rho^{2}, \frac{1}{\rho^{2}} D^{2} \psi_{0}\right]+\frac{1}{\rho^{3}} \frac{\partial}{\partial z}\left[K\left(\psi_{0}-\frac{U}{2} \rho^{2}\right)\right]^{2}=0,
$$

and since it can be shown that

$$
J\left[\varphi, \frac{1}{\rho^{2}} K^{\prime}(\varphi) K(\varphi)\right]=\frac{1}{\rho^{3}} \frac{\partial}{\partial z}[K(\varphi)]^{2},
$$

for some field $\varphi$, we can write

$$
J\left[\psi_{0}-\frac{U}{2} \rho^{2}, \frac{1}{\rho^{2}}\left\{D^{2} \psi_{0}+K^{\prime}\left(\psi_{0}-\frac{U}{2} \rho^{2}\right) K\left(\psi_{0}-\frac{U}{2} \rho^{2}\right)\right\}\right]=0 .
$$

Therefore we have

$$
D^{2} \psi_{0}+K^{\prime}\left(\psi_{0}-\frac{U}{2} \rho^{2}\right) K\left(\psi_{0}-\frac{U}{2} \rho^{2}\right)=\rho^{2} Z\left(\psi_{0}-\frac{U}{2} \rho^{2}\right),
$$

for some function $Z(\varphi)$. We note that the function $Z$ can be related to the pressure head $H\left(\psi_{0}-U \rho^{2} / 2\right)=p_{0}+\left|\mathbf{u}_{0}\right|^{2} / 2$ using Bernoulli's principle (Batchelor 2000, Section 7.5) by $Z=H^{\prime}$.

We can now determine $Z$ outside the vortex using the same method as was used to determine $K$. In the far field $D^{2} \psi_{0} \rightarrow 0$ hence we have

$$
-\frac{1}{2} \epsilon^{2} k^{2} U=Z\left(-\frac{U}{2} \rho^{2}\right)
$$

so $Z$ is the constant function

$$
Z=-\frac{1}{2} U \epsilon^{2} k^{2},
$$


where we recall that $k U=1$. For the flow inside the vortex we follow Moffatt (1969) and take

$$
H(\varphi)=H_{0}+\lambda \varphi \quad \Longrightarrow \quad Z(\varphi)=\lambda,
$$

for some constant $\lambda$. Therefore our leading order streamfunction satisfies

$$
\left[D^{2}+\epsilon^{2} k^{2}\right] \psi_{0}= \begin{cases}0 & r>a, \\ \left(\lambda+\frac{1}{2} U \epsilon^{2} k^{2} \rho^{2}\right) \rho^{2} & r<a,\end{cases}
$$

such that $\psi_{0}=U \rho^{2} / 2$ on $r=a$ and $\partial \psi_{0} / \partial r$ is continuous across $r=a$ so the velocity is continuous across the vortex boundary. These boundary conditions will allow us to uniquely determine $\lambda$. Once we have solved for $\psi_{0}$, we can find $w_{0}$ from $\psi_{0}$ using Eq. (2.19).

We observe from Eq. (2.30) that there are two natural lengthscales in the problem - the vortex radius, $a$, and the wavelength $(\epsilon k)^{-1}$. We therefore expect our solution to consist of an interior vortex region with $\epsilon k r \ll 1$ and an exterior wavefield where $\epsilon k r \sim 1$. We begin by determining the interior vortex solution and then determine the exterior wavefield, the wave amplitude is obtained by matching to the interior solution.

\section{Interior vortex solution}

Here we solve for the leading order streamfunction, $\psi_{0}$, in the interior region around the vortex where $\epsilon k r \ll 1$.

\subsection{The flow inside the vortex}

Inside the vortex, where $r \leqslant a$, we have

$$
\left[D^{2}+\epsilon^{2} k^{2}\right] \psi_{0}=\left(\lambda+\frac{1}{2} U \epsilon^{2} k^{2} \rho^{2}\right) \rho^{2},
$$

from Eq. (2.30), with general solution

$$
\psi_{0}=\left[\frac{\lambda}{\epsilon^{2} k^{2}}+\frac{U}{2}\right] r^{2} \sin ^{2} \theta+r \sin \theta \sum_{m=1}^{\infty} \mathrm{P}_{m}^{1}(\cos \theta)\left[A_{m} \mathrm{j}_{m}(\epsilon k r)+B_{m} \mathrm{y}_{m}(\epsilon k r)\right], \quad r \leqslant a .
$$

Here the $\mathrm{P}_{n}^{1}$ are associated Legendre polynomials and the $\mathrm{j}_{n}$ and $\mathrm{y}_{n}$ are spherical Bessel functions of the first and second kind respectively and we have imposed the conditions that our solution is $2 \pi$ periodic and $\theta=0, \pi$ are streamlines. Since we require $\psi_{0}$ to be non-singular at the origin we must have $B_{m}=0$ for all $m$ and the condition that $\psi_{0}=U \rho^{2} / 2$ at $r=a$ gives that $A_{m}=0$ for $m \geqslant 2$ and

$$
A_{1}=-\frac{\lambda a}{\epsilon^{2} k^{2} \mathrm{j}_{1}(\epsilon k a)} \text {. }
$$

Therefore our solution is

$$
\psi_{0}=\left(\frac{U}{2}+\frac{\lambda}{\epsilon^{2} k^{2}}\left[1-\frac{a \mathrm{j}_{1}(\epsilon k r)}{r \mathrm{j}_{1}(\epsilon k a)}\right]\right) r^{2} \sin ^{2} \theta, \quad r \leqslant a,
$$

where the value of $\lambda$ is determined by the continuity of polar velocity, $u_{\theta}$, across $r=a$.

\subsection{The flow around the vortex}

Outside the vortex, for $r \geqslant a$, we have

$$
\left[D^{2}+\epsilon^{2} k^{2}\right] \psi_{0}=0
$$


from Eq. (2.30) with general solution

$$
\psi_{0}=r \sin \theta \sum_{m=1}^{\infty} \mathrm{P}_{m}^{1}(\cos \theta)\left[C_{m} \mathrm{j}_{m}(\epsilon k r)+D_{m} \mathrm{y}_{n}(\epsilon k r)\right], \quad r \geqslant a .
$$

Requiring that $\psi_{0}=U \rho^{2} / 2$ on $r=a$ and our solution decays away from the origin for $\epsilon k r \ll 1$ gives that

$$
\psi_{0}=\frac{U a}{2} \frac{\mathrm{y}_{1}(\epsilon k r)}{\mathrm{y}_{1}(\epsilon k a)} r \sin ^{2} \theta, \quad r \geqslant a .
$$

\subsection{The full interior solution}

Our final condition on $\psi_{0}$ is that the polar velocity, $u_{\theta 0}$, is continuous across $r=a$ which gives that $\partial \psi_{0} / \partial r$ must be continuous. Using Eq. (3.7) and Eq. (3.4) we have

$$
\frac{\lambda a}{\epsilon^{2} k^{2}} \frac{\epsilon k a \mathrm{j}_{2}(\epsilon k a)}{\mathrm{j}_{1}(\epsilon k a)}=-\frac{U a}{2} \frac{\epsilon k a \mathrm{y}_{2}(\epsilon k a)}{\mathrm{y}_{1}(\epsilon k a)}
$$

so

$$
\lambda=-\frac{15 U}{2 a^{2}}\left(1-\frac{38}{105}(\epsilon k a)^{2}+O\left(\epsilon^{4}\right)\right) .
$$

Noting that $\epsilon k r \ll 1$ for $r \leqslant a$ and neglecting terms of order $O\left(\epsilon^{4}\right)$ and higher, we can expand Eq. (3.4) as

$$
\psi_{0}=\frac{U}{2}\left(1+\left[\frac{3}{2}-\frac{19}{35}(\epsilon k a)^{2}\right]\left[1-\frac{r^{2}}{a^{2}}\right]+\frac{3(\epsilon k a)^{2}}{280}\left[9-\frac{14 r^{2}}{a^{2}}+\frac{5 r^{4}}{a^{4}}\right]\right) r^{2} \sin ^{2} \theta, \quad r \leqslant a .
$$

In the limit of $\epsilon k a \rightarrow 0$ this result reduces to the classical vortex solution of Hill (1894) given by

$$
\psi_{0}=\frac{3 U}{4}\left[\frac{5}{3}-\frac{r^{2}}{a^{2}}\right] r^{2} \sin ^{2} \theta, \quad r \leqslant a .
$$

Similarly, expanding Eq. (3.7) for $\epsilon k r \ll 1$ and neglecting terms of order $O\left(\epsilon^{4}\right)$ or higher we obtain

$$
\psi_{0}=\frac{U a^{3}}{2}\left[1+\frac{(\epsilon k a)^{2}}{2}\left(\frac{r^{2}}{a^{2}}-1\right)\right] \frac{\sin ^{2} \theta}{r}, \quad r \geqslant a,
$$

which reduces to inviscid flow around a sphere in the case of $\epsilon k a \rightarrow 0$.

\section{Exterior wave field}

We now determine the wave field in the exterior region where $\epsilon k r \sim 1$. From Eq. (2.30) we have

$$
\left[D^{2}+\epsilon^{2} k^{2}\right] \psi_{0}=0
$$

for $r>a$. This equation may alternatively be derived from the linearised Euler equations under the assumption that far from the vortex the wave wake is small in amplitude (Johnson \& Crowe 2021) showing that the linear wave field satisfies the nonlinear system. Since solutions to Eq. (4.1) will vary over a lengthscale of order $O\left(\epsilon^{-1}\right)$ we now balance the two terms in this equation by introducing the long radial scale $R=\epsilon r$. Switching to spherical coordinates gives

$$
\frac{\partial^{2} \psi_{0}}{\partial R^{2}}+\frac{\sin \theta}{R^{2}} \frac{\partial}{\partial \theta}\left[\frac{1}{\sin \theta} \frac{\partial \psi_{0}}{\partial \theta}\right]+k^{2} \psi_{0}=0,
$$


which can be solved using separation of variables for solution

$$
\psi_{0}=k R \sum_{n=1}^{\infty} \sin \theta \mathrm{P}_{n}^{1}(\cos \theta)\left[A_{n} \mathrm{j}_{n}(k R)+B_{n} \mathrm{y}_{n}(k R)\right] .
$$

Long (1953) observed that waves are not seen ahead of objects moving through a rotating flow. These observations are supported by the theoretical work of McIntyre (1972) for the case of an unbounded rotating flow motivating us to use the radiation condition of no upstream waves. Fraenkel (1956) showed that this condition can be satisfied by $B_{n}=0$ for $n \geqslant 2$ and

$$
A_{n}= \begin{cases}0, & n \text { odd } \\ -\frac{(2 n+1)(n-2) !}{2^{n}\left(\frac{n}{2}+1\right) !\left(\frac{n}{2}-1\right) !} B_{1}, & n \text { even }\end{cases}
$$

so our solution may be written as

$$
\psi_{0}=B R \sin \theta\left[\mathrm{y}_{1}(k R) \sin \theta+\sum_{n=1}^{\infty} \frac{(4 n+1)(2 n-2) !}{2^{2 n}(n+1) !(n-1) !} \mathrm{j}_{2 n}(k R) \mathrm{P}_{2 n}^{1}(\cos \theta)\right],
$$

where $B=-k B_{1}$ can be found by matching to the interior solution. We note that spherical Bessel functions take on a trigonometric form for $k R=O(1)$ justifying the definition of $k=1 / U$ as a wavenumber. Substituting $r=R / \epsilon$, we can see that wavelength of these waves is $O(1 / \epsilon)$ assuming that $U$ remains $O(1)$.

In order to find the form of $B$ we match Eq. (4.5) to the inner solution in Eq. (3.7) by substituting $R=\epsilon r$. Noting that

$$
\mathrm{j}_{n}(x) \sim \frac{x^{n}}{(2 n+1) ! !} \quad \text { and } \quad \mathrm{y}_{1}(x) \sim-\frac{1}{x^{2}},
$$

for small argument $x$ we have

$$
\psi_{0} \sim \epsilon B \mathrm{y}_{1}(\epsilon k r) r \sin ^{2} \theta+O\left(\epsilon^{4}\right) .
$$

Hence, by comparison with Eq. (3.7), we have

$$
B=\frac{U a}{2 \epsilon \mathrm{y}_{1}(\epsilon k a)}=-\frac{\epsilon U a^{3} k^{2}}{2}+O\left(\epsilon^{3}\right),
$$

to leading order in $\delta$. We note that close to the vortex $(R \ll 1), \psi_{0}$ is dominated by the $\mathrm{y}_{1}(k R)$ term. This behaviour is consistent with the expected dipole structure near the vortex. Additionally, since $B=O(\epsilon)$, the wave amplitude is $O(\epsilon)$ and hence small for $R=O(1)$.

\section{Summary of leading order solution}

Before moving on to the solvability conditions required to determine the slow time evolution of the vortex, we present the full leading order solution. The interior Hill's vortex solution is given by

$$
\psi_{0}=\left\{\begin{array}{lr}
\frac{U}{2}\left(1+\left[\frac{3}{2}-\frac{19}{35}(\epsilon k a)^{2}\right]\left[1-\frac{r^{2}}{a^{2}}\right]+\frac{3(\epsilon k a)^{2}}{280}\left[9-\frac{14 r^{2}}{a^{2}}+\frac{5 r^{4}}{a^{4}}\right]\right) r^{2} \sin ^{2} \theta, & r \leqslant a, \\
\frac{U a^{3}}{2}\left(1+\frac{(\epsilon k a)^{2}}{2}\left[\frac{r^{2}}{a^{2}}-1\right]\right) \frac{\sin ^{2} \theta}{r}, & r \geqslant a .
\end{array}\right.
$$

for $\epsilon k r \ll 1$. The terms of order $O\left((\epsilon k a)^{2}\right)$ in this result correspond to both the near field inertial waves and the modification of internal vortex structure by the rotation. Far 


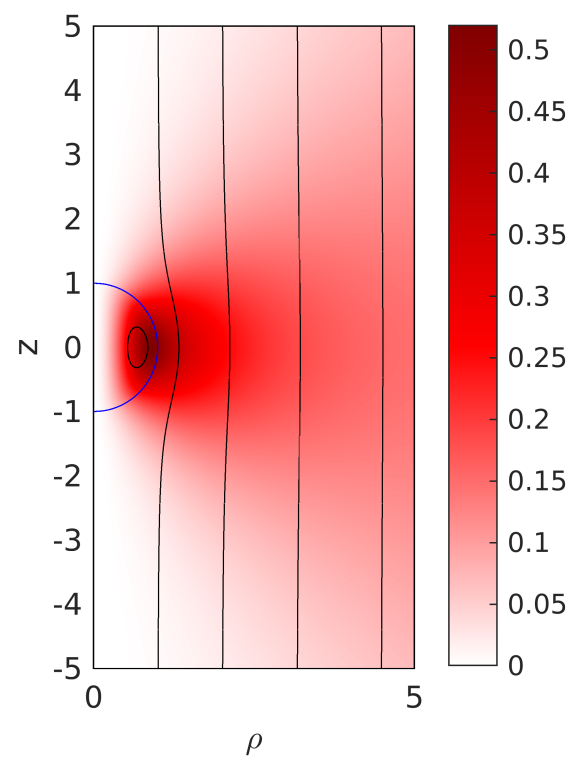

(a)

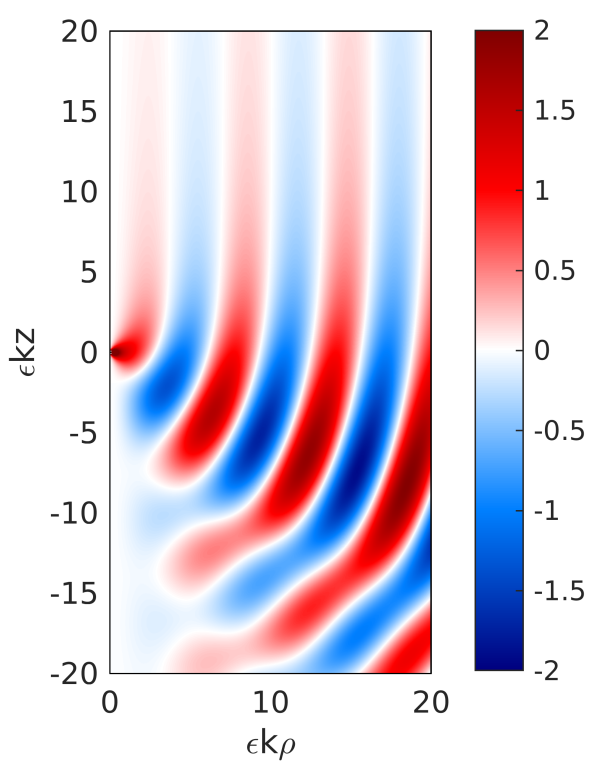

(b)

Figure 2: (a) Plot of $\psi_{0}$ from Eq. (5.1) for $U=1, a=1$ and $\epsilon=02$. The black lines denote streamlines of $\psi_{0}^{\prime}=\psi_{0}-U \rho^{2} / 2$ and the blue line denotes the vortex boundary, $r=a$. (b) Plot of $\psi_{0} /|B|$ from Eq. (5.2) as a function of the long spatial scales $(\epsilon k \rho, \epsilon k z)=$ $k R(\sin \theta, \cos \theta)$.

from the vortex, the exterior wave field streamfunction is

$$
\psi_{0}=\frac{a U R \sin \theta}{2 \epsilon \mathrm{y}_{1}(\epsilon k a)}\left[\mathrm{y}_{1}(k R) \sin \theta+\sum_{n=1}^{\infty} \frac{(4 n+1)(2 n-2) !}{2^{2 n}(n+1) !(n-1) !} \mathrm{j}_{2 n}(k R) \mathrm{P}_{2 n}^{1}(\cos \theta)\right],
$$

for long radial scale $R=\epsilon r$. We note that the amplitude of the wave field is $O(\epsilon)$ since $\mathrm{y}_{1}(\epsilon k a)=O\left(\epsilon^{-2}\right)$.

Fig. 2.(a) shows the streamfunction, $\psi_{0}$, for the interior vortex solution from Eq. (5.1) for $U=1, a=1$ and $\epsilon=0.2$. The black lines denote streamlines in a frame moving with the vortex - corresponding to streamlines of $\psi_{0}^{\prime}=\psi_{0}-U \rho^{2} / 2$. The blue line denotes the streamline $\psi_{0}^{\prime}=0$ so corresponds to the vortex boundary, $r=a$. The streamlines of $\psi_{0}^{\prime}$ describe the particle paths of incoming fluid over the short timescale, $t$, and will vary over the slow scale, $T$, as the vortex speed and radius change. Fig. 2.(b) shows the streamfunction, $\psi_{0}$, for the exterior wave field given by Eq. (5.2). We plot $\psi_{0} /|B|$ (for $B$ defined in Eq. (4.8)) as a function of the long spatial scales $(\epsilon k \rho, \epsilon k z)=k R(\sin \theta, \cos \theta)$ so the plotted result is unchanged for all values of $\epsilon, U$ and $a$. We observe a wave wake in the region $z<0$ with decaying upstream perturbation. The wave amplitude is maximal along the line $\theta=2 \pi / 3$ as discussed in Appendix B.

\section{Solvability conditions}

We now consider the energy balance and azimuthal vorticity to determine solvability conditions which will allow us to examine the long-time decay of the vortex. 


\subsection{Conservation of energy}

The leading order Kinetic energy of the vortex is given by

$$
E_{0}=\frac{1}{2} \int\left(u_{\rho 0}^{2}+u_{z 0}^{2}+w_{0}^{2}\right) \mathrm{d} V=\int \frac{1}{2 \rho^{2}}\left(\left|\nabla \psi_{0}\right|^{2}+\epsilon^{2} k^{2} \psi_{0}^{2}\right) \mathrm{d} V,
$$

where the integral is taken over the interior region. The generation of the wave field removes energy from the vortex leading to vortex decay with the loss of vortex energy balancing the outward flux of wave energy.

We can show (see Appendix A) that the total energy flux is given by

$$
\delta \frac{d E_{0}}{d T}=U \int\left\{\frac{1}{2 \rho^{2}}\left[\left(\frac{\partial \psi_{0}}{\partial z}\right)^{2}-\left(\frac{\partial \psi_{0}}{\partial \rho}\right)^{2}\right] \hat{\boldsymbol{z}} \cdot \hat{\boldsymbol{n}}+\frac{1}{\rho^{2}} \frac{\partial \psi_{0}}{\partial \rho} \frac{\partial \psi_{0}}{\partial z} \hat{\boldsymbol{\rho}} \cdot \hat{\boldsymbol{n}}\right\} \mathrm{d} S,
$$

where the right-hand integral is evaluated over the surface of a sphere of radius $r=R / \epsilon$ and is independent of $R$. The required expression for $\psi_{0}$ on the sphere is given by the far-field streamfunction, Eq. (5.2), however as the wave energy flux is $R$ independent we can use the asymptotic form of Eq. (5.2) for small $R$ given by

$$
\psi_{0}=\frac{\epsilon U a^{3}}{2}\left[\frac{\sin ^{2} \theta}{R}+\frac{k^{4} R^{3} \sin ^{2} \theta \cos \theta}{8}\right]+O\left(R^{4}\right),
$$

to simplify calculation of Eq. (6.2). Evaluation of Eq. (6.2) gives that

$$
\delta \frac{d E_{0}}{d T}=-\frac{\pi \epsilon^{4} k^{4} U^{3} a^{6}}{4}+O\left(R^{4}\right)
$$

where the $O\left(R^{4}\right)$ term may be set to zero as the result is independent of $R$. Noting that $k=1 / U$, defining $\delta=\epsilon^{4}$ and calculating $E_{0}$ as the leading order kinetic energy of a Hill's vortex,

$$
E_{0}=\frac{10 \pi U^{2} a^{3}}{7}
$$

our final result for the loss of vortex energy to the wave field is

$$
\frac{d}{d T}\left[\frac{10 \pi U^{2} a^{3}}{7}\right]=-\frac{\pi a^{6}}{4 U} .
$$

\subsection{Conservation of centre vorticity}

As the vortex decays, we expect vortical fluid to escape and hence we do not necessarily expect all vortex streamlines to form closed surfaces correct to $O(\delta)$. We proceed by following the peak vorticity approach (Flierl \& Haines 1994; Johnson \& Crowe 2021) and assuming that the innermost stream-surface of the streamfunction in a frame moving with the vortex

$$
\psi^{\prime}=-\frac{U}{2} \rho^{2}+\psi
$$

remains closed to $O(\delta)$. The vorticity

$$
\omega_{\phi}=-\frac{1}{\rho} D^{2} \psi
$$

contained within this streamsurface is assumed to be conserved. Here this innermost streamsurface corresponds to a torus of vanishing minor axis about the maximum of $\psi^{\prime}$ so all quantities within this streamsurface can be evaluated at the position of this maximum, referred to from now on as the 'vortex centre'. 
To leading order we have

$$
\omega_{\phi}=\frac{15 U \rho}{2 a^{2}},
$$

and as the maximum of $\psi^{\prime}$ occurs for $r=a / \sqrt{2}, \theta=\pi / 2$ we have that the vorticity within the innermost streamsurface is

$$
\omega_{\phi c}=\frac{15 U}{2 \sqrt{2} a} .
$$

Taking this quantity to be constant to $O(\delta)$ gives that

$$
\frac{d}{d T}\left[\frac{U}{a}\right]=0,
$$

hence $U / a$ is equal to its initial value, $U\left(T_{0}\right) / a\left(T_{0}\right)$, at $T=T_{0}$. We note that this approach may also be thought of as assuming that the value of $\omega_{\phi}$ is conserved following a particle within the innermost stream-surface.

\section{Vortex decay}

By combining the loss of vortex energy to the wave field, Eq. (6.6), with the conservation of centre vorticity, Eq. (6.11), we have

$$
a(T)=a_{0} \exp \left[-\frac{7 a_{0}^{3}}{200 U_{0}^{3}}\left(T-T_{0}\right)\right],
$$

and

$$
U(T)=U_{0} \exp \left[-\frac{7 a_{0}^{3}}{200 U_{0}^{3}}\left(T-T_{0}\right)\right],
$$

where $(U, a)=\left(U_{0}, a_{0}\right)$ at some initial time $T=T_{0}$ corresponding to $t=t_{0}=\epsilon^{-4} T_{0}$.

To leading order in $\epsilon$, the maximum of $\psi^{\prime}$ within the vortex occurs for $r=a / \sqrt{2}$ and $\theta=\pi / 2$ and is given by

$$
\psi_{c}=\frac{3 U a^{2}}{16}
$$

where we use a subscript ' $c$ ' to denote the value of a quantity at the vortex centre. Using Eq. (7.1) and Eq. (7.2) we can write

$$
\psi_{c}=\frac{3 U_{0} a_{0}^{2}}{16} \exp \left[-\frac{21 a_{0}^{3}}{200 U_{0}^{3}}\left(T-T_{0}\right)\right],
$$

which may be tested against numerical simulations. Additionally, the centre vorticity may be written using Eq. (6.10) as

$$
\omega_{\phi c}=\frac{15 U_{0}}{2 \sqrt{2} a_{0}}
$$

which clearly remains independent of time by the assumption of conservation of centre vorticity. Finally the leading order (in $\epsilon$ ) vortex energy should decay as

$$
E_{0}=\frac{10 \pi U_{0}^{2} a_{0}^{3}}{7} \exp \left[-\frac{7 a_{0}^{3}}{40 U_{0}^{3}}\left(T-T_{0}\right)\right] .
$$




\section{Numerical simulations}

To test the model presented here we run numerical simulations of the nondimensional, axisymmetric governing equations (see Eq. (2.2)) using the Dedalus package (Burns et al. 2020). Simulations are run for rotation parameters $\epsilon \in\{0,0.1,0.2,0.3,0.4,0.5\}$. We solve the axisymmetric Navier-Stokes equations on the domain $(\rho, z) \in[0,51.2] \times[-51.2,51.2]$ with 1024 gridpoints in the $\rho$ direction and 2048 gridpoints in the $z$ direction. Fields are expanded in terms of Fourier modes in $z$ and Chebyshev polynomials in $\rho$ and integrated for $t \in[0,50]$ using a second order semi-implicit BDF (backwards differentiation formula) scheme with a timestep of $2 \times 10^{-3}$. Due to the choice of basis functions, our fields satisfy periodic boundary conditions in the $z$ direction and we impose rigid wall conditions $\left(u_{\rho}=0\right)$ on $\rho=51.2$. The boundary conditions at $\rho=0$ are set by multiplying all equations through by the highest power of $\rho$ and substituting $\rho=0$. This is dealt with internally by Dedalus and efficiently deals with the coordinate singularity.

Since we do not know $U(t)$ in advance, numerical simulations are performed in coordinates moving at speed 1 in the $z$ direction by setting $U=1$ in Eq. (2.2a). Therefore a vortex moving with speed $U \approx 1$ should remain near the centre of the domain and a vortex found to be moving in the negative $z$ direction has $U<1$. Viscous diffusion with a viscosity of $\nu=10^{-4}$ is included for numerical stability. This diffusion acts to damp instabilities which would otherwise form near the rear stagnation point (Moffatt \& Moore 1978; Pozrikidis 1986; Protas \& Elcrat 2016) leading to a breakdown of the vortex.

The $\rho$ and $z$ velocity components, $\left(u_{\rho}, u_{z}\right)$, are initialised using the classical Hill's vortex solution (corresponding to the $\epsilon=0$ limit of $\psi_{0}$ in Eq. (5.1)) with $U=1$ and $a=1$ while the swirl velocity $w$ is set by

$$
w=-\frac{\epsilon k}{2 \rho} \psi_{0} .
$$

At early times we do not expect to see the vortex decay predicted in Section 7 since this requires an established wave field. Instead we expect fast transient behaviour where the wave field is formed and the vortex adjusts to the effects of rotation. Once the transient adjustment near the vortex has abated, comparison with our theory can be made. We therefore begin all comparison from $t_{0}=10$ which we observe to be sufficient for the wave-field to develop and the vortex to adjust. It should be noted that the vortex speed and radius may change due this transient adjustment phase and hence the values of $U\left(t_{0}\right)=U_{0}$ and $a\left(t_{0}\right)=a_{0}$ may differ from the values of $U=a=1$ imposed at $t=0$.

Fig. 3 shows the streamfunction $\psi$ as a function of $(\rho, z)$ for $\epsilon=0.4$ and $t \in\{0,25,50\}$. The formation of the wave-field can be clearly seen with waves forming behind the vortex of wavelength $\lambda=2 \pi U / \epsilon \approx 16$. Small additional disturbances are seen in the wake of the vortex resulting from the initial transient evolution and from fluid escaping the vortex core.

A supplementary movie (Movie.mp4) shows the temporal evolution of the velocity fields for $\epsilon=0.4$ - we opt to plot the velocity fields since they show the wave propagation more clearly than the streamfunction. Due to the fast decay of $u_{\rho}$ and $u_{z}$ with $\rho$, we plot $\rho u_{\rho}$ and $\rho u_{z}$ instead so that the wave field is visible for large $\rho$. We observe a wave field of similar structure to the prediction shown in Fig. 2.(b) with no disturbances propagating upstream. At later times the vortex appears to move downwards, corresponding to a velocity, $U$, of less than 1 . Additionally, a small disturbance left by the initial adjustment of the vortex can be seen travelling downwards. Due to the periodicity of the domain in the $z$ direction and the boundary at $\rho=51.2$ acting to reflect outgoing waves, we might expect waves to loop around the domain and interact with the vortex. While some 


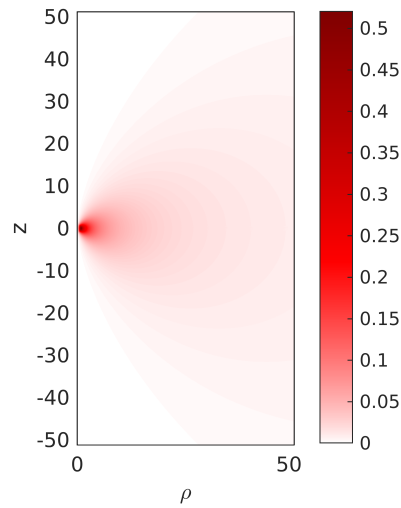

(a)

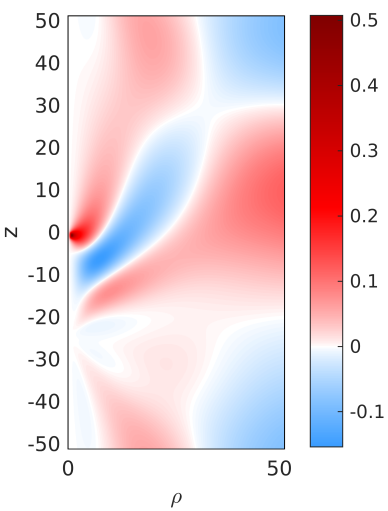

(b)

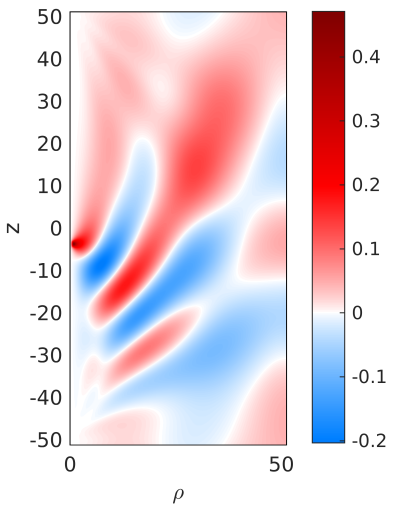

(c)

Figure 3: The streamfunction, $\psi$, from numerical simulations with $\epsilon=0.4$. Results are shown for (a) $t=0$, (b) $t=25$ and (c) $t=50$.

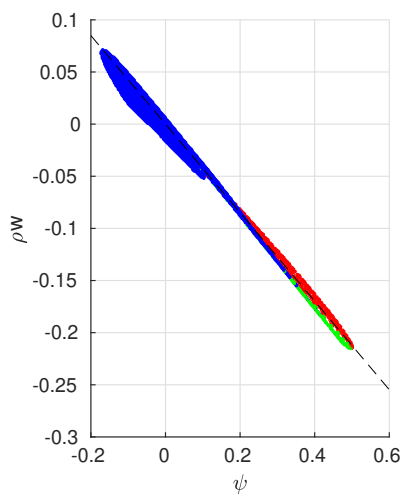

(a)

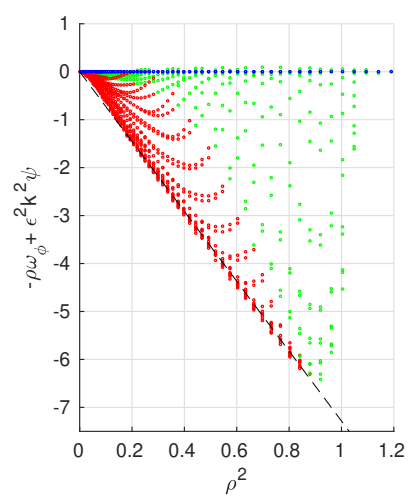

(b)

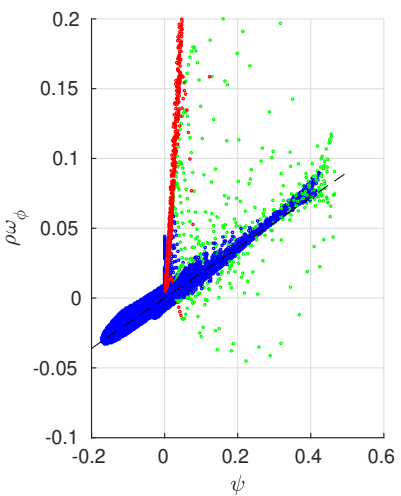

(c)

Figure 4: Scatter plots for $\epsilon=0.4$ and $t=30$ showing (a) $\rho w$ against $\psi$, (b) $-\rho \omega_{\phi}+\epsilon^{2} k^{2} \psi$ against $\rho^{2}$ and (c) $\rho \omega_{\phi}$ against $\psi$. The dashed lines give our theoretical predictions.

disturbances are seen re-entering the domain, the simulation stop time, $t=50$, is seen to be sufficient to prevent these waves from reaching the vortex.

To leading order in $\delta$ we expect that the swirl velocity, $w$, both inside and outside the vortex satisfies

$$
\rho w=K(\psi)=-\epsilon k \psi,
$$

from Eq. (2.19) and Eq. (2.22). Similarly the vorticity and streamfunction are related by

$$
-\rho \omega_{\phi}+\epsilon^{2} k^{2} \psi= \begin{cases}0, & r>a, \\ -\frac{15 U}{2 a^{2}}\left[1-\frac{3}{7}(\epsilon k a)^{2}\right] \rho^{2}, & r<a,\end{cases}
$$

using Eq. (2.30), Eq. (3.9) and $-\rho \omega_{\phi}=D^{2} \psi$. If these relations are satisfied throughout the vortex evolution then the vortex will remain close to a Hill's vortex and our theory should be valid. These relations tested are for $\epsilon=0.4$ and $t=30$ in Fig. 4 using scatter plots. These plots show the numerical values of two quantities at some point in $(\rho, z)$ space; each point represents a different numerical gridpoint and all gridpoints are 
included. Points are coloured - red points represent points within the vortex $(r<0.9 a)$, green points represent points near the vortex edge $(0.9 a \leqslant r \leqslant 1.1 a)$ and blue points represent points outside the vortex $(r>1.1 a)$. Theoretical predictions using Eq. (8.2) and Eq. (8.3) are included as dashed lines.

Fig. 4(a) shows the angular momentum, $\rho w$, against the streamfunction, $\psi$. We can see that all points lie very close to a line of constant gradient so Eq. (8.2) remains accurate late in the evolution. The value of $-\epsilon k$ can be determined by fitting the points to a straight line. Fig. 4(b) shows $-\rho \omega_{\phi}+\epsilon^{2} k^{2} \psi$ against $\rho^{2}$ with a focus on the region close to the vortex, $\rho \leqslant 1$. Points close to the centre of the vortex lie close to our prediction (see Eq. (8.3)) however we note that the predicted relation does not hold near the edge of the vortex where viscosity acts to smooth the discontinuity in vorticity. Our prediction is determined using the fitted value of $\epsilon k$ to determine $U=1 / k$ and the method described below to estimate $a$. Finally Fig. 4(c) shows $\rho \omega_{\phi}$ against $\psi$ which, by Eq. (8.3), we expect to be linearly related outside the vortex. As expected we observe that the blue points (corresponding to points outside the vortex) lie close to a line of gradient $\epsilon^{2} k^{2}$.

While viscosity acts to maintain the vortex integrity by damping small scale instabilities, it also leads to a loss of around $10 \%$ of the domain integrated energy. These viscous effects are particularly pronounced near the vortex edge as seen in Fig. 4 since Hill's vortex has a vorticity discontinuity across $r=a$. The smoothing of this discontinuity makes it particularly difficult to determine an accurate value for the vortex radius, $a$, while the drag associated with the boundary layers can modify the vortex speed, $U$.

To account for viscous effects, we assume that a diagnosed quantity, $f$, is a function of the linear wave drag with coefficient $\epsilon^{4}$ and the viscous damping with coefficient $\nu$. If both $\epsilon^{4}$ and $\nu$ are small, we may expand

$$
f\left(\epsilon^{4}, \nu, t\right)=f(0,0, t)+\epsilon^{4} \frac{\partial f}{\partial \epsilon^{4}}(0,0, t)+\nu \frac{\partial f}{\partial \nu}(0,0, t)+O\left(\epsilon^{8}, \epsilon^{4} \nu, \nu^{2}\right),
$$

and hence we may determine the effect of the wave drag by running a simulation with $\epsilon=0$ and subtracting the results from a non-zero $\epsilon$ run to get

$$
f\left(\epsilon^{4}, \nu, t\right)-[f(0, \nu, t)-f(0,0, t)]=f(0,0, t)+\epsilon^{4} \frac{\partial f}{\partial \epsilon^{4}}(0,0, t)+O\left(\epsilon^{8}, \epsilon^{4} \nu, \nu^{2}\right),
$$

where $f(0,0, t)=f(0,0,0)$ is constant in time and known from the analytical Hill's vortex solution or equivalently the initial conditions. We now use this method to estimate the maximum streamfunction, $\psi_{c}$, and vortex energy, $E_{0}$, adjusting for viscosity. The unadjusted value of $E_{0}$ is determined by integrating $\psi \omega_{\phi}$ over the region $r<a$ (see Eq. (A 10)) where the value of $a$ is estimated as the radial distance from the centre of the vortex to the point where the vorticity is reduced to $10 \%$ of its maximum value.

Fig. 5 shows the centre streamfunction, $\psi_{c}$, centre vorticity, $\omega_{\phi c}$, and vortex energy, $E_{0}$. Here the centre streamfunction, $\psi_{c}$, is the maximum value of $\psi^{\prime}$ (defined in Eq. (6.7)) inside the vortex and the centre vorticity, $\omega_{\phi c}$, is the value of $\omega_{\phi}$ at the position where $\psi^{\prime}=\psi_{c}$. As stated above, we perform all comparisons from $t=t_{0}=10$ onwards; we also normalise all quantities by their value at $t_{0}$. Solid lines denote data from our numerical simulations and dashed lines denote our predictions from Section 7. Due to the difficulty in determining the radius and speed of the adjusted vortex to the accuracy required to calculate $\left(a_{0} / U_{0}\right)^{3}$ at $t=t_{0}$, we instead take $a_{0} / U_{0}=1.15$ by fitting the predictions for $\psi_{c}$ to the numerical data in $t \in[10,20]$. We expect the value of $a_{0} / U_{0}$ to depend on $\epsilon$ so perform a fitting for all value of $\epsilon$ and find that all values satisfy $a_{0} / U_{0} \approx 1.15$. It should be noted that this value of $a_{0} / U_{0}$ lies within the range of possible values calculated using 


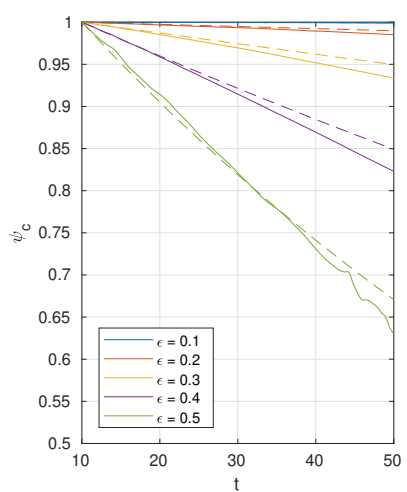

(a)

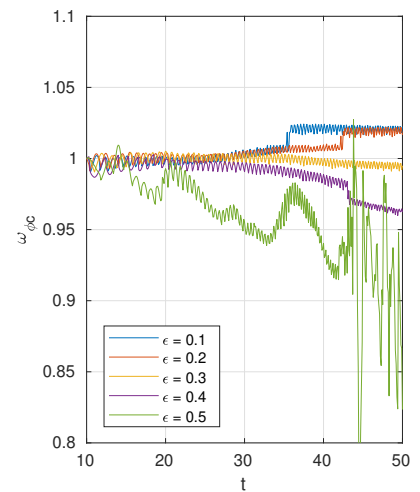

(b)

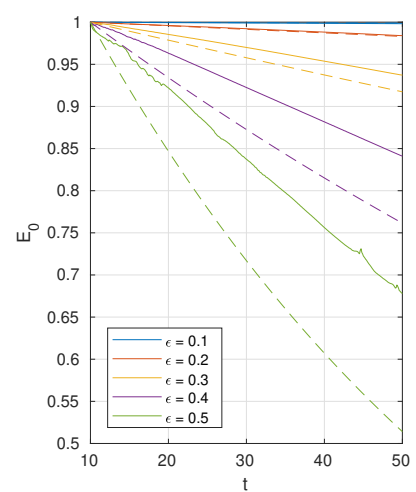

(c)

Figure 5: (a) the centre streamfunction, $\psi_{c}$, (b) the centre vorticity, $\omega_{\phi c}$, (c) the vortex energy, $E_{0}$. All quantities are normalised by their value at $t=10$ and shown from $t=10$ for all non-zero $\epsilon$ values simulated.

different methods for determining the vortex speed and radius so is consistent with our numerical data.

Fig. 5(a) shows the comparison between the numerical and theoretical values of the centre streamfunction, $\psi_{c}$. We observe close agreement between the prediction and the theory though, as noted previously, some fitting was involved to account for the difficulties in determining $a_{0} / U_{0}$. As the results are consistent over the full range of $\epsilon$, we conclude that the time scaling $t \sim O\left(1 / \epsilon^{4}\right)$ is likely correct. Fig. 5(b) shows the centre vorticity, $\omega_{\phi c}$, from our numerical simulations. We observe that $\omega_{\phi c}$ is conserved to within $3 \%$ throughout the evolution for $\epsilon \leqslant 0.4$ which is consistent with our assumption that the centre vorticity is conserved throughout the evolution. We do observe larger, oscillatory deviations for $\epsilon=0.5$ which may suggest the presence of wavelike oscillations inside the vortex due to the stronger rotation - these phenomena are likely to be strongly nonlinear and may act to modify the vortex decay.

Fig. 5(c) shows a comparison between the vortex energy, $E_{0}$, from simulations and theory. We note that our predictions are less accurate for the larger $\epsilon$ values and this discrepancy may be due to several factors. Firstly the difficulties in determining the vortex radius are particularly important here due to the integration domain depending on $a$. Secondly, the expression for $E_{0}$ does not include the rotational component of the energy, $w^{2}$, as this is an $O\left(\epsilon^{2}\right)$ contribution. However, our numerical simulations suggest that this component may be significant in the finite $\epsilon$ case and may even increase with time due to finite $\epsilon$ effects which could be related to conservation of angular momentum. Including this component would increase the predicted value back towards the numerical value. Finally, the neglected higher order components of the translational kinetic energy $T=|\nabla \psi|^{2} /\left(2 \rho^{2}\right)$, and the wave drag will modify the energy balance in the finite $\epsilon$ case.

\section{Discussion and conclusions}

We have used an asymptotic analysis to examine the decay of a Hill's vortex in a weakly rotating flow. We observe that the effect of rotation is to allow the emission of inertial waves which carry energy away from the vortex leading to its decay. This energy flux is small, on the order of $O\left(1 / R o^{4}\right)=(2 \Omega L / V)^{4}$, resulting in a slow decay scale on the order of $t \sim R o^{4}$ for large Rossby number $R o=V /(2 \Omega L)$. By considering the energy balance 
and combining this result with the conservation of centre vorticity (Flierl \& Haines 1994; Johnson \& Crowe 2021) we derived results for the evolution of the vortex speed and radius under the assumption that the vortex remains a Hill's vortex to leading order. The present approach is based on the existence of a 'Long's model' for this geometry, i.e. a linear integral of the full nonlinear equations of motion, and the wavefield here satisfies that linear equation. An example where this does not apply is the monopolar vortex of (Griffiths 1999), where significant nonlinear effects can occur in an intermediate region between the vortex and wavefield.

Replacing the dimensional scales in Eqs. (7.1) and (7.2) we find that the (dimensional) vortex radius, $a$, and translation speed, $U$, decay as

$$
(a, U)=\left(a_{0}, U_{0}\right) \exp \left[-\frac{14}{25}\left(\frac{\Omega a_{0}}{U_{0}}\right)^{4} \frac{U_{0}}{a_{0}}\left(t-t_{0}\right)\right],
$$

where $a_{0}$ and $U_{0}$ are the (dimensional) radius and velocity of the vortex at $t=t_{0}$. These results could be similarly derived using the equivalent wave drag approach of Johnson \& Crowe (2021) as outlined in Appendix C.

Numerical simulations have been used to test our predictions and show good agreement in the case of small inverse Rossby number. For larger values of $\epsilon=1 / R o$ we observe some discrepancies between the theory and numerics likely due to the modification of the vortex structure by finite $\epsilon$ effects. For order $O(1)$ values of $\epsilon$ the vortex does not remain spherical throughout the evolution and becomes an oblate spheroid, this may be related to the conservation of angular momentum arising from the non-zero swirl velocity induced by rotation. The decay of an initially spherical vortex in a strongly rotating fluid is therefore not likely to be analytically tractable so would have to be examined numerically.

\subsection{Extensions}

The problem considered here can be extended in several ways. Firstly, Hill's vortex is one particular vortex from the doubly infinite family examined by Moffatt (1969). In the far field, all vortices in this family appear as dipoles of the same strength therefore the linear wavefield and wave energy flux will be unchanged from the Hill's vortex case. Similarly, the dependence of the energy and centre vorticity on $a$ and $U$ will be the same for all member of this vortex family with the only change being the value of coefficient in the expression for $E_{0}$. Therefore we may use Eqs. (6.2) and (6.11) for a Moffatt vortex with

$$
E_{0}=\beta \pi U^{2} a^{3}
$$

to get that the (dimensional) radius and speed to decay as

$$
(a, U)=\left(a_{0}, U_{0}\right) \exp \left[-\frac{4}{5 \beta}\left(\frac{\Omega a_{0}}{U_{0}}\right)^{4} \frac{U_{0}}{a_{0}}\left(t-t_{0}\right)\right],
$$

where $(a, U)=\left(a_{0}, U_{0}\right)$ at $t=t_{0}$. We note that the Hill's vortex result corresponds to the case where $\beta=10 / 7$.

Another extension of this problem would be to consider the decay of a spherical vortex in a domain bounded in the radial direction. Taking the boundary to be a distance of order $1 / \epsilon$ from the vortex, the leading order vortex solution will be unchanged however the wave-field, which varies over the scale $R=1 / \epsilon$, will be discretised by the wall boundary conditions. While the rigid wall would result in wave reflection, we do not expect significant re-absorption of energy as most waves would be reflected far behind 
the vortex. This modified wave-field would lead to a modified energy flux and hence change the decay rate of the vortex. Finally, we could examine the decay of vortex rings travelling along the rotation axis of a weakly rotating fluid using a combined numerical and analytic approach. This will be a topic for future work and may be useful for estimating the lifespan of these typically long-lived structures in a rotating flow.

Supplementary material. A supplementary movie is available at ...

Funding. This work was funded by the UK Natural Environment Research Council under grant number NE/S009922/1.

Declaration of Interests. The authors report no conflict of interest.

\section{Appendix A. Energy flux}

The kinetic energy density is given by

$$
E=\frac{1}{2}|\mathbf{u}|^{2}
$$

and satisfies

$$
\left[\delta \frac{\partial}{\partial T}-U \frac{\partial}{\partial z}\right] E+\nabla \cdot[\mathbf{u}(E+p)]=0 .
$$

We now integrate Eq. (A 2) over a sphere of radius $r=R / \epsilon$ for $R=O(1$ ) (denoted $V$ ) and consider the leading order contribution in $\epsilon$ from each term. Noting that the wave-field is $O(\epsilon)$ for $r \sim O(1 / \epsilon)$ we can neglect the terms cubic in velocity and set the pressure using the linearised $z$-momentum equation

$$
-U \frac{\partial u_{z}}{\partial z}=-\frac{\partial p}{\partial z}
$$

so $p=U u_{z}$. The leading order volume integrated energy balance can now be written as

$$
\delta \frac{d}{d T} \int E \mathrm{~d} V=U \int\left[E \hat{\boldsymbol{z}} \cdot \hat{\boldsymbol{n}}-u_{z} \mathbf{u} \cdot \hat{\boldsymbol{n}}\right] \mathrm{d} S,
$$

where the integral on the right-hand side is evaluated over the surface of $V$ (denoted $\partial V)$. Substituting

$$
\mathbf{u} \cdot \hat{\boldsymbol{n}}=-\frac{1}{\rho} \frac{\partial \psi}{\partial z} \hat{\boldsymbol{\rho}} \cdot \hat{\boldsymbol{n}}+\frac{1}{\rho} \frac{\partial \psi}{\partial \rho} \hat{\boldsymbol{z}} \cdot \hat{\boldsymbol{n}}
$$

and

$$
E=\frac{1}{2}\left(\frac{1}{\rho^{2}}|\nabla \psi|^{2}+w^{2}\right)
$$

gives

$$
\delta \frac{d}{d T} \int E \mathrm{~d} V=U \int\left\{\frac{1}{2 \rho^{2}}\left[\left(\frac{\partial \psi}{\partial z}\right)^{2}-\left(\frac{\partial \psi}{\partial \rho}\right)^{2}+\rho^{2} w^{2}\right] \hat{\boldsymbol{z}} \cdot \hat{\boldsymbol{n}}+\frac{1}{\rho^{2}} \frac{\partial \psi}{\partial \rho} \frac{\partial \psi}{\partial z} \hat{\boldsymbol{\rho}} \cdot \hat{\boldsymbol{n}}\right\} \mathrm{d} S .
$$

The energy, $E$, within $V$ may now be split into an interior vortex component and an exterior wave component. Using simple scalings with $\epsilon$, we may show that the interior vortex component dominates for small $\epsilon$. Hence, for all $R$, the leading order kinetic energy contained with in $V$ is given by $E_{0}$, the kinetic energy of Hill's vortex. Therefore, the surface flux term on the right-hand side of Eq. (A 7) must also be independent of $R$. 
Noting that $\psi=O(\epsilon)$ and $w=O\left(\epsilon^{2}\right)$ on $\partial V$ we may neglect the $w$ terms to get our final leading order result

$$
\delta \frac{d E_{0}}{d T}=U \int\left\{\frac{1}{2 \rho^{2}}\left[\left(\frac{\partial \psi_{0}}{\partial z}\right)^{2}-\left(\frac{\partial \psi_{0}}{\partial \rho}\right)^{2}\right] \hat{\boldsymbol{z}} \cdot \hat{\boldsymbol{n}}+\frac{1}{\rho^{2}} \frac{\partial \psi_{0}}{\partial \rho} \frac{\partial \psi_{0}}{\partial z} \hat{\boldsymbol{\rho}} \cdot \hat{\boldsymbol{n}}\right\} \mathrm{d} S .
$$

The vortex energy, $E_{0}$, to leading order in $\epsilon$ is given by

$$
E_{0}=\int \frac{1}{2 \rho^{2}}\left|\nabla \psi_{0}\right|^{2} \mathrm{~d} V=\frac{1}{2} \int\left[\nabla \cdot\left(\frac{\psi_{0} \nabla \psi_{0}}{\rho^{2}}\right)-\frac{\psi_{0}}{\rho^{2}}\left(\nabla^{2} \psi_{0}-\frac{2}{\rho} \frac{\partial \psi_{0}}{\partial \rho}\right)\right] \mathrm{d} V,
$$

so neglecting the small surface term gives

$$
E_{0}=-\frac{1}{2} \int \frac{1}{\rho^{2}} \psi_{0} D^{2} \psi_{0} \mathrm{~d} V=\pi \int \psi_{0} \omega_{\phi 0} \mathrm{~d} \rho \mathrm{d} z
$$

hence

$$
E_{0}=\int_{r \leqslant a} \frac{15 \pi U^{2}}{2 a^{2}}\left(\frac{5}{4}-\frac{3 r^{2}}{4 a^{2}}\right) r^{4} \sin ^{3} \theta \mathrm{d} r \mathrm{~d} \theta=\frac{10 \pi U^{2} a^{3}}{7} .
$$

The leading order kinetic energy in the outer wave field is given by

$$
E_{w}=\frac{1}{2 \rho^{2}}\left(\left|\nabla \psi_{0}\right|^{2}+\epsilon^{2} k^{2} \psi_{0}^{2}\right)
$$

so the total wave energy within a region of radius $r \geqslant O(1 / \epsilon)$ scales as

$$
\int E_{w} \mathrm{~d} V \sim \frac{\left\langle\psi_{0}\right\rangle^{2}}{r}+\epsilon^{2} r\left\langle\psi_{0}\right\rangle^{2}
$$

where $\left\langle\psi_{0}\right\rangle=O(\epsilon)$ is the $\epsilon$ scaling of the wave field streamfunction, $\psi_{0}$. Taking $r=R / \epsilon$ gives the result that the wave energy scales as

$$
\int E_{w} \mathrm{~d} V \sim O\left(\epsilon^{3}\right)
$$

which is much smaller than the contribution from the vortex energy as noted above. However, taking $r=R / \epsilon^{4}$ gives an order 1 energy contribution from the swirl component. This suggests that the energy lost from the vortex is stored in the wave field over a scale of $r=O\left(1 / \epsilon^{4}\right)$. This is unsurprising since, assuming order 1 propagation speeds, this scale also describes the distance travelled by a wave over the timescale, $T$. Therefore the wave field is confined to the region $r \leqslant O\left(1 / \epsilon^{4}\right)$ which suggests the existence of an ultra far-field region for $r \gg 1 / \epsilon^{4}$ in which there is no disturbance.

\section{Appendix B. The Taylor (1922) solutions}

Dotting Eq. (2.2a) with $\mathbf{u}$ gives the energy equation

$$
\frac{\partial E}{\partial t}+\nabla \cdot \mathbf{F}=0
$$

where $E=\mathbf{u}^{2} / 2$ and $\mathbf{F}=(-U \hat{\mathbf{z}}+\mathbf{u}) E+\mathbf{u} p$. Integrating Eq. (B 1) over a volume $V$ with surface $S$ fixed in the frame moving at speed $U$ gives the energy conservation equation

$$
\frac{\mathrm{d}}{\mathrm{d} t} \int_{V} E \mathrm{~d} V=-\oint_{S}[-E U \hat{\mathbf{z}} \cdot \hat{\mathbf{n}}+(p+E) \mathbf{u} \cdot \hat{\mathbf{n}}] \mathrm{d} S .
$$

The vortex energy decreases at a rate given by the rate of working of the pressure force and the flux of energy through the boundary. In terms of the polar angle $\theta$ and azimuthal 


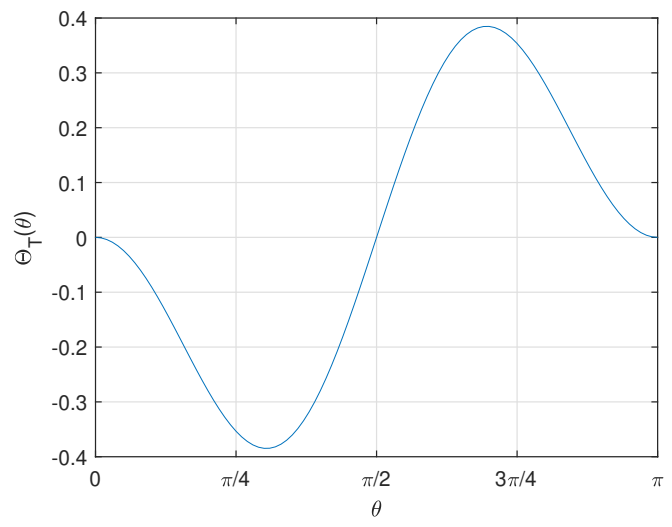

(a)

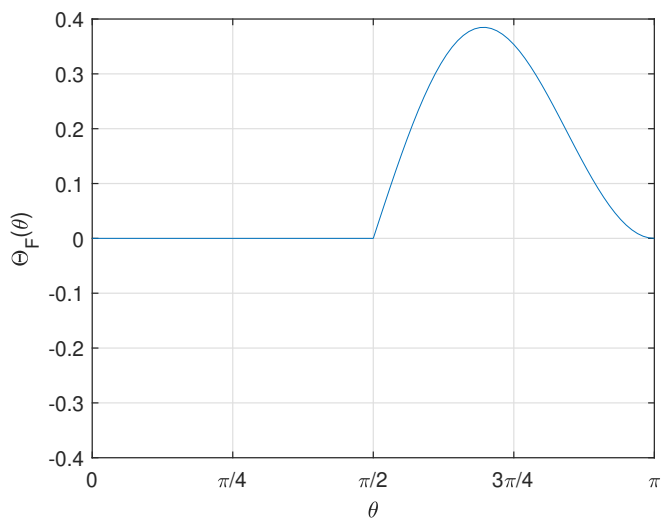

(b)

Figure 6: The directional dependence of the outward energy fluxes in terms of the polar angle, $\theta$. (a) Taylor (1922)-type solutions, $\Theta_{\mathrm{T}}(\theta)$. (b) Fraenkel (1956)-type solutions, $\Theta_{\mathrm{F}}(\theta)$.

angle $\phi$ the flux integral for the energy travelling outwards across a sphere of radius $R$ is thus

$$
\begin{aligned}
F_{R}=R^{2} \int_{0}^{2 \pi} \int_{0}^{\pi}[-E U \cos \theta+(p & \left.+E) u_{r}\right] \sin \theta \mathrm{d} \theta \mathrm{d} \phi \\
& =2 \pi R^{2} \int_{0}^{\pi}\left[-E U \cos \theta+(p+E) u_{r}\right] \sin \theta \mathrm{d} \theta .
\end{aligned}
$$

As in the main text, the velocities appearing in Eq. (B 3) are relative to the laboratory frame. Taylor (1922) takes the same moving frame but considers velocities relative to the moving frame. Thus the streamfunction in Taylor (1922) is related to that in the text here through $\psi_{T}=-\left[U r^{2} \sin ^{2} \theta\right] / 2+\psi$. Taylor looks for solutions with form $\psi=U f(r) \sin ^{2} \theta$. For large $R$ this form of solution gives

$$
F_{R}=U^{3} f_{1}(R) \int_{0}^{\pi} \Theta_{\mathrm{T}}(\theta) \mathrm{d} \theta
$$

where $\Theta_{\mathrm{T}}(\theta)=-\sin ^{3} \theta \cos \theta$, the contribution to the outward flux along a ray $\theta=$ constant, and $f_{1}(R)$ follows from $f(r)$ as

$$
f_{1} \sim \frac{1}{2}\left[f^{\prime 2}+\frac{4 \Omega^{2}}{U^{2}} f^{2}\right] \rightarrow \frac{4 \Omega^{2} C}{U^{2}}, \quad \text { as } \quad r \rightarrow \infty,
$$

for some constant $C$. Taylor discusses in detail the particular solution whose tangential and normal velocity components vanish on the sphere $r=a$. For this solution $C=$ $1+3 R o^{2}+9 R o^{4}$ where $R o=U /(2 \Omega a)$. For the Taylor solutions of Scase \& Terry (2018), chosen to match a Hill's vortex in $r<a, C=1$.

Fig. 6(a) shows the directional dependence $\Theta_{\mathrm{T}}(\theta)$ of the outward energy flux for solutions of the Taylor type. The integral in Eq. (B 4), i.e. the area under the graph in Fig. 6(a), vanishes, as it must in order that no work is done on the enclosed fluid and the motion is steady. This however gives an inward flux of energy from $z>0$, i.e. $0<\theta<\pi / 2$ to balance the outward flux in $z<0$, i.e. $\pi / 2<\theta<\pi$ and thus is unsatisfactory for problems without an energy source at infinity. This feature is distinct from upstream influence: the modification of the oncoming flow by columnar modes which propagate 
upstream from the disturbance and do satisfy the radiation condition (Lighthill 1967; McIntyre 1972; Scase \& Terry 2018).

The Fraenkel (1956) solutions used in Eq. (5.2) for the wavefield contain extra terms, odd in $\theta-\pi / 2$, that in the far-field precisely cancel $\Theta_{\mathrm{T}}(\theta)$ for $0<\theta<\pi / 2$ and exactly reinforce it for $\pi / 2<\theta<\pi$, giving the $\theta$ dependence

$$
\Theta_{F}(\theta)= \begin{cases}0, & 0<\theta<\pi / 2, \\ \Theta_{\mathrm{T}}(\theta), & \pi / 2<\theta<\pi\end{cases}
$$

(see Fig. 6(b)) and a maximum contribution along the line $\theta=2 \pi / 3$, as apparent in Fig. 2.(b). The unsteadiness and finite forcing time of the flow widen the peak response in the unsteady simulations of Fig. 3.

\section{Appendix C. Wave drag approach}

An alternative approach to deriving the rate of decay of the vortex energy is to compute the rate of working of the vortex on the fluid (Johnson \& Crowe 2021).

The far-field inertial wave wake satisfies the homogeneous equation

$$
\psi_{\rho \rho}-(1 / \rho) \psi_{\rho}+\psi_{z z}+\kappa^{2} \psi=0 \text { for } \rho>0,
$$

where $\kappa=\epsilon k$. On this scale the vortex appears as a dipole of strength $\mu=2 \pi U a^{3}$ at the origin, so

$$
\psi=-U a^{3} \delta(z) \quad \text { on } \quad \rho=0 .
$$

The solution to (C 1), (C 2) has the Fourier representation

$$
\psi(\rho, z)=\frac{1}{2 \pi} \int_{-\infty}^{\infty} \hat{\psi}(\rho, \lambda) \exp (\mathrm{i} \lambda z) \mathrm{d} \lambda
$$

where, for outward group velocity (inward phase velocity), $\hat{\psi}$ is given by

$$
\hat{\psi}=-U a^{3} \begin{cases}\left(\lambda^{2}-\kappa^{2}\right)^{1 / 2} \rho \mathrm{K}_{1}\left[\left(\lambda^{2}-\kappa^{2}\right)^{1 / 2} \rho\right], & \lambda^{2}>\kappa^{2} \\ (\mathrm{i} \pi / 2)(\operatorname{sgn} \lambda)\left(\kappa^{2}-\lambda^{2}\right)^{1 / 2} \rho \mathrm{H}_{1}^{(1)}\left[(\operatorname{sgn} \lambda)\left(\kappa^{2}-\lambda^{2}\right)^{1 / 2} \rho\right], & \lambda^{2}<\kappa^{2} .\end{cases}
$$

Here $K_{1}$ is the modified Bessel function of the second kind of order one and $H_{1}^{(1)}$ is the Hankel function of the first kind of order one. Fraenkel (1956) gives a closely related result. Inverting (C 4) gives the wave field in Eq. (5.2). This is however unnecessary and the energy flux is obtained below directly from (C 4).

To derive the drag force, let $\xi\left(\rho_{0}, z\right)$ be the displacement from the radius $\rho_{0}$ of a particle originally at a distance $\rho=\rho_{0}$ from the rotation axis far upstream. Then the drag $D$ exerted on the vortex by the fluid is given by the component of the pressure force in the negative- $z$ direction on the material surface $\rho=\rho_{0}+\xi\left(\rho_{0}, z\right)$,

$$
D=-2 \pi \rho \int_{-\infty}^{\infty} p \frac{\partial \xi}{\partial z} \mathrm{~d} z
$$

Substituting $u_{\rho}=-U \partial \xi / \partial z$ from the definition of $\xi$, and $p=U u_{z}$ from the linearised $z$-momentum equation gives

$$
D=2 \pi \rho \int_{-\infty}^{\infty} u_{\rho} u_{z} \mathrm{~d} z=-\frac{2 \pi}{\rho} \int_{-\infty}^{\infty} \frac{\partial \psi}{\partial z} \frac{\partial \psi}{\partial \rho} \mathrm{d} z=-\frac{1}{\rho} \int_{-\infty}^{\infty} \frac{\widehat{\partial \psi}_{\partial z}}{\partial \rho} \widehat{\partial \psi}^{*} \mathrm{~d} \lambda
$$

from Plancherel's theorem with * denoting the complex conjugate. The drag determination $D$ in $(\mathrm{C} 6)$ is independent of the radius $\rho$ along which the integral is evaluated and 
thus it is convenient to let $\rho \rightarrow \infty$, so

$$
\begin{aligned}
& \frac{\widehat{\partial \psi}}{\partial z} \rightarrow \mathrm{i} \lambda U a^{3}(\mathrm{i} \pi / 2) \omega \rho|2 / \pi \omega \rho|^{1 / 2} \exp [\mathrm{i}(\omega \rho-3 \pi / 4)], \\
& \frac{\partial \psi}{\partial \rho} \rightarrow U a^{3}(\mathrm{i} \pi / 2) \omega^{2} \rho|2 / \pi \omega \rho|^{1 / 2} \exp [\mathrm{i}(\omega \rho-\pi / 4)],
\end{aligned}
$$

for $|\lambda|<\kappa$ and exponentially small otherwise, with $\omega=(\operatorname{sgn} \lambda)\left(\kappa^{2}-\lambda^{2}\right)^{1 / 2}$. Then

$$
D=\frac{\pi}{2} U^{2} a^{6} \int_{-\kappa}^{\kappa}|\lambda|\left(\kappa^{2}-\lambda^{2}\right) \mathrm{d} \lambda=\pi U^{2} a^{6} \kappa^{4} / 4 .
$$

As expected, this is positive showing that the fluid exerts a decelerating drag on the vortex.

The terms involving the energy density $E$ in (B 1) are cubic in small quantities and so the leading order energy flux is given by the quadratic flux of pressure with the flux of energy radially outwards from the vortex becoming

$$
W=2 \pi \rho \int_{-\infty}^{\infty} u_{\rho} p \mathrm{~d} z=2 \pi \rho \int_{-\infty}^{\infty} U u_{\rho} u_{z} \mathrm{~d} z=U D=\frac{\pi \epsilon^{4} a^{6}}{4 U},
$$

the rate of working of the vortex on the fluid through the drag force. The vortex energy equation

$$
\frac{\mathrm{d} E_{0}}{\mathrm{~d} T}=-W
$$

matches (6.6).

\section{REFERENCES}

Batchelor, G. K. 2000 An Introduction to Fluid Dynamics. Cambridge University Press.

Bretherton, F. P. 1967 The time-dependent motion due to a cylinder moving in an unbounded rotating or stratified fluid. J. Fluid Mech. 28 (3), 545-570.

Burns, K. J., Vasil, G. M., Oishi, J. S., Leconnet, D. \& Brown, B. P. 2020 Dedalus: A flexible framework for numerical simulations with spectral methods. Phys. Rev. Res. 2, 023068.

Flierl, G. R. \& Haines, K 1994 The decay of modons due to Rossby wave radiation. Phys. Fluids 6 (10), 3487-3497.

Ford, R., McIntyre, M. E. \& Norton, W. A. 2000 Balance and the slow quasimanifold: Some explicit results. Journal of the Atmospheric Sciences 57 (9), 1236-1254.

Fraenkel, L. E. 1956 On the flow of rotating fluid past bodies in a pipe. Proc. R. Soc. A. 233 (1195), 506-526.

Fraenkel, L. E. 1972 Examples of steady vortex rings of small cross-section in an ideal fluid. J. Fluid Mech. 51 (1), 119-135.

GRIfFIths, R. M. 1999 The interaction between vorticity and internal gravity waves. PhD thesis, University of Cambridge.

Hill, M. J. M. 1894 On a spherical vortex. Phil. Trans. R. Soc. (A.) 185, 213-245.

Johnson, E. R. \& Crowe, M. N. 2021 The decay of a dipolar vortex in a weakly dispersive environment. J. Fluid Mech. p. in press.

LighthiLl, M. J. 1967 On waves generated in dispersive systems by travelling forcing effects, with applications to the dynamics of rotating fluids. J. Fluid Mech. 27 (4), 725-752.

Llewellyn Smith, S. G. \& Ford, R. 2001 Three-dimensional acoustic scattering by vortical flows. II. Axisymmetric scattering by Hill's spherical vortex. Phys. Fluids 13 (10), 2890 2900.

LONG, R. R. 1953 Steady motion around a symmetrical obstacle moving along the axis of a rotating liquid. J. Met. 10, 197-203. 
Machicoane, N., Labarre, V., Voisin, B., Moisy, F. \& Cortet, P.-P. 2018 Wake of inertial waves of a horizontal cylinder in horizontal translation. Phys. Rev. Fluids 3, 034801.

MCInTyre, M. E. 1972 On Long's hypothesis of no upstream influence in uniformly stratified or rotating flow. J. Fluid Mech. 52 (2), 209-243.

Moffatt, H. K. 1969 The degree of knottedness of tangled vortex lines. J. Fluid Mech. 35 (1), $117-129$.

Moffatt, H. K. \& Moore, D. W. 1978 The response of Hill's spherical vortex to a small axisymmetric disturbance. J. Fluid Mech. 87 (4), 749-760.

Norbury, J. 1972 A steady vortex ring close to Hill's spherical vortex. Proc. Cam. Phil. Soc. 72 (2), 253-284.

Norbury, J. 1973 A family of steady vortex rings. J. Fluid Mech. 57 (3), 417-431.

PozRikidis, C. 1986 The nonlinear instability of Hill's vortex. J. Fluid Mech. 168, 337-367.

Protas, B. \& Elcrat, A. 2016 Linear stability of Hill's vortex to axisymmetric perturbations. J. Fluid Mech. 799, 579-602.

Scase, M. M. \& Terry, H. L. 2018 Spherical vortices in rotating fluids. J. Fluid Mech. 846, R4.

TAYlor, G. I. 1922 The motion of a sphere in a rotating liquid. Proc. R. Soc. A 102 (715), 180-189. 